\title{
A Run-Time Method Based on Observable Data for the Quality Assessment of GNSS Positioning Solutions
}

\author{
Fabio Dovis, Bilal Muhammad, Ernestina Cianca, and Khurram Ali
}

\begin{abstract}
Several location-aware applications rely on the position estimated by means of Global Navigation Satellite Systems (GNSS), which are known to estimate an accurate position in an open environment. However, the quality of the estimated position is degraded in harsh environments in terms of accuracy and reliability. Liability-critical services, such as location-based charging, transportation, and road tolling, are threatened by the use of an unreliable position of the user, and the level of trust in the estimated position has to be considered to avoid a failure of the full service chain. Such an issue is faced by means of integrity monitoring methods in the field of GNSS. However, when dealing with harsh environments, integrity monitoring techniques designed for aeronautical applications would lead to very conservative results and to the rejection of all the positions obtained. Such conservative approach is based on the theoretical error models for the estimation of the pseudorange standard deviation in open sky. The purpose of this work is to propose an innovative method for estimating the pseudorange standard deviation extrapolating it from measurements of observable data, to assess the confidence level in the obtained positions in relation to the real environment surrounding the user. While measuring the pseudorange standard deviation taking into account environment conditions and receiver accuracy, the user is able to achieve better estimation of the user equivalent range error (UERE). Estimating the UERE from raw pseudorange measurements with the proposed run-time method and its subsequent use in the computation of protection levels using the receiver autonomous integrity monitoring (RAIM) algorithm shows significant improvement in navigation system availability by deriving tight protection levels.
\end{abstract}

Index Terms-GNSS, RAIM, Integrity, UERE, GPS positioning.

\section{INTRODUCTION}

$\mathbf{T}$ HE quality of the estimated position depends on the ability of the receiver to perform the measurement, as well as on the error sources affecting the signal and also on the propagation environment. Furthermore, the assessment of the reliability of the obtained position is essential for a wide range of appli-

Manuscript received August 15, 2014; revised December 7, 2014; accepted January 11, 2015. Date of publication May 6, 2015; date of current version October 19, 2015.

F. Dovis is with the Department of Electronics and Telecommunications, Politecnico di Torino, Turin 10129, Italy (e-mail: fabio.dovis@ polito.it).

B. Muhammad and E. Cianca are with the Department of Electronic Engineering, University of Rome Tor Vergata, Rome 00133, Italy (e-mail: bilal@ ing.uniroma2.it; ernestina.cianca@uniroma2.it).

K. Ali is with the Department of Electrical Engineering, COMSATS Institute of Information Technology, 54000 Lahore, Pakistan (e-mail: khurram.ali@ciitlahore.edu.pk).

Color versions of one or more of the figures in this paper are available online at http://ieeexplore.iee. org.

Digital Object Identifier 10.1109/JSAC.2015.2430513 cations where the accuracy error might not be extremely small but must be bounded in order to avoid failures of the full service chain. Pseudorange standard deviation is a key parameter used to estimate the confidence intervals of the positioning solution that are used to understand the reliability of the received signal and for the prediction of the accuracy of position. The scope of this paper is to present a run-time method that, on the basis of observed pseudorange measurements, is able to provide an estimate of the actual pseudorange accuracy, without relying on a priori models, which may lead to over-restrictive conditions for the solution acceptability. Knowledge of the pseudorange error is a key point for the definition of reliability concepts that can be used in environments (e.g., urban) where the classical definition of the integrity used in aeronautics is too restrictive. Harsh propagation conditions and poor satellite visibility are the common scenarios, and on top of that, the "as is" application of the integrity methodology would cause the exclusion of the totality of the obtained position, thus degrading the navigation system availability. One of the reasons of this result is the mismatch of the error models used in the user equivalent range error (UERE) [1] estimation and the urban environment [2]. According to the classical definition, the pseudorange standard deviation is defined at the system level, taking into account the contributions of the different error sources. UERE is generally considered independent and identically distributed (i.i.d.) for all the satellites, and it represents the residual error after the removal of all the predictable error components (e.g., by using correction models). Such a residual error is then modeled as an additive random variable that is Gaussian, with zero mean and standard deviation $\sigma_{\text {UERE }}$. Under such hypotheses, the standard deviation of the position error $\sigma_{\text {pos }}$ can be estimated [3] as

$$
\sigma_{\mathrm{pos}}=\mathrm{DOP} \cdot \sigma_{\mathrm{UERE}}
$$

where DOP is the dilution of precision, which is a coefficient depending on the geometry of the navigation satellites as seen by the user performing the measurement.

Many models were recently proposed in order to predict the pseudorange measurement using series statistics and autoregressive models [4]. Modeling the pseudorange in a proper way allows to compute better positioning [5] by identifying and considering only error-free measured values. Nevertheless, this modeling based on observed samples does not aim to estimate the error statistics but to predict the future pseudorange value. In [6], the single contribution of the measurement of pseudorange is analyzed and, where possible, measured or 
better estimated. In [7], Belabbas et al. measured the instantaneous pseudorange error (IPRE) as a sum of contributions of the error sources. These contributions are computed as a difference between estimated and reference values with oneyear observation data received in several locations worldwide. The work highlights how considering a common and fixed standard deviation for any pseudorange can be quite a restricting approximation. Nevertheless, IPRE is evaluated still using the concept of contributions of errors, and it needs IGS station postprocessed data.

This work proposes a new approach to the problem of the estimation of $\sigma_{\text {UERE }}$ by using the raw measured values of pseudoranges (i.e., without application of corrections at the receiver level) in quasi-real time by using a regular navigation receiver. The work aims at providing an algorithm that can be used with commercial receivers, thus processing measurements that are affected by all the error sources along the reception and processing chain. In this way, the pseudorange standard deviation is assessed from the "point of view" of the user, and not relying, for instance, on residual error models that are, by nature, representing the average behavior of the environment. This new concept of UERE estimation, on one hand, provides a method of assessing the user environment; on the other hand, it lays down the foundations for more elaborated processing for the estimation of the reliability of the positioning solution in environments (e.g., urban environment), which cannot be properly represented by the classical method of pseudorange error estimation.

This paper is organized as follows. Section II recalls the main aspects related to the classical UERE definition and highlights the inherent conservatism in the classical UERE method. Section III introduces the procedure for the run-time estimation discussing the implementation of the strategies to de-trend the data from the satellite movement and the observation sampling time to assure the processing of uncorrelated values. Section IV will then discuss the use of the estimated run-time UERE with respect to classical UERE in the assessment of the confidence in the obtained solution and the service availability. The conclusion is drawn in Section V.

\section{Classical Method of Pseudorange RESIDUAL ERROR ESTIMATION}

The design of GNSS integrity is based on the assumption that the pseudorange measurement residual errors are characterized by zero-mean Gaussian distribution. However, real-world error distribution are not always characterized by zero-mean Gaussian distribution, which would invalidate the assumption of zero-mean Gaussian distribution, consequently risking the reliability of GNSS integrity design. This problem is dealt with by overbounding the actual pseudorange residual error distribution with an overbounded zero-mean Gaussian distribution [8], such that

$$
\begin{gathered}
\phi_{o}(z) \leq \phi_{a}(z) \forall z \leq 0 \\
\phi_{o}(z) \geq \phi_{a}(z) \forall z>0 .
\end{gathered}
$$

$\phi_{a}$ is the cumulative distribution function (cdf) of the actual error distribution, and $\phi_{o}$ is the cdf of the overbounded zero-mean
Gaussian distribution. Taking the root sum square of the variance of overbounding distribution of individual pseudorange measurement error sources, the $1 \sigma$ UERE [1] for each satellite in view can be obtained as

$$
\sigma_{\text {UERE }, \mathrm{i}}=\sqrt{\sigma_{\text {clk\&eph }, \mathrm{i}}^{2}+\sigma_{\mathrm{iono}, \mathrm{i}}^{2}+\sigma_{\text {tropo }, \mathrm{i}}^{2}+\sigma_{\mathrm{mp}, \mathrm{i}}^{2}+\sigma_{\mathrm{recv}, \mathrm{i}}^{2}} .
$$

$\sigma_{\text {UERE, } i}$ is the standard deviation of the pseudorange measurement error experienced by a GNSS receiver after the application of correction models on the signal received from the $i$ th satellite. Neglecting the subscript ' $\mathrm{i}$ ' for clarity, $\sigma_{\text {clk\&eph }}$ is the standard deviation of the satellite clock and orbit residual error, $\sigma_{\text {iono }}$ is the standard deviation of single-frequency residual errors due to ionosphere, $\sigma_{\text {tropo }}$ is the standard deviation of residual error due to troposphere, $\sigma_{\mathrm{mp}}$ is the standard deviation of error due to multipath, and $\sigma_{\text {recv }}$ is the standard deviation of error due to receiver thermal noise, interference, and receiver imperfections.

\section{A. Nominal Pseudorange Residual Error Models}

The pseudorange residual error estimation models, mainly developed for applications such as civil aviation, are equally applicable to the urban environment with exception to multipath [9]. These models are briefly discussed as follows.

- Satellite clock and ephemeris residual error standard deviation is generally referred to as user range accuracy (URA) [10]. The URA index is provided to the users of Global Positioning System (GPS) within the navigation message. The most common value of GPS URA is $2.4 \mathrm{~m}$ [11].

- Standard deviation of residual error due to ionosphere is estimated by the GPS users as in [12] using

$$
\sigma_{\text {iono }}=F_{p p} \cdot \max \left(\Delta_{\text {iono, } 20 \%}, \tau_{\mathrm{ver}}\right)
$$

where $\Delta_{\text {iono }}, 20 \%$ is the $20 \%$ of the total delay caused due to ionosphere, $F_{p p}$ is the obliquity factor, and $\tau_{\mathrm{ver}}$ is the minimum vertical delay:

$$
\begin{aligned}
\Delta_{\text {iono }, 20 \%} & =0.2 \cdot c \cdot T_{\text {iono, ver }} \\
\tau_{\text {vert }} & = \begin{cases}9 \mathrm{~m}, & 0^{\circ} \leq\left|\phi_{m}\right| \leq 20^{\circ} \\
4.5 \mathrm{~m}, & 20^{\circ}<\left|\phi_{m}\right| \leq 55^{\circ} \\
6 \mathrm{~m}, & 55^{\circ}<\left|\phi_{m}\right| \leq 90^{\circ} .\end{cases}
\end{aligned}
$$

$\mathrm{T}_{\text {iono,ver }}$ is the delay due to ionosphere computed using the Klobuchar model [13], $\tau_{\text {ver }}$ is the minimum vertical delay, $\phi_{m}$ is the geomagnetic latitude in degree, and c is the speed of light in vacuum.

- Residual error due to troposphere is modeled [12] by

$$
\sigma_{\text {tropo }}=\sigma_{\mathrm{TVE}} \cdot m(\mathrm{El})
$$

where $\sigma_{\mathrm{TVE}}=0.12$, and $m(E l)$ is the mapping function valid for satellite at elevation angle $\mathrm{El}>4^{\circ}$ :

$$
m(E l)=\frac{1.001}{\sqrt{0.002001+\sin ^{2} \mathrm{El}}} .
$$

- Code tracking error mainly due to receiver thermal noise depends on the design characteristics of the 


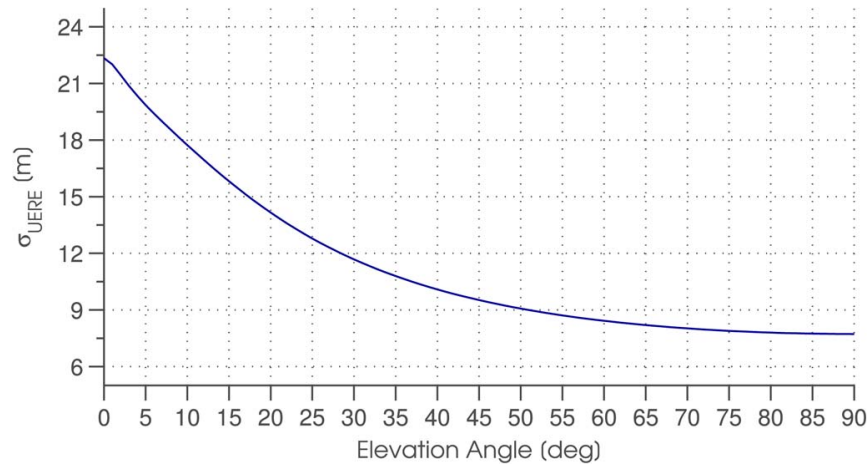

Fig. 1. 1 $\sigma$ UERE budget as a function of satellite elevation angle.

receiver and signal modulation. The tracking error due to thermal noise of the aviation receiver is modeled as a function of satellite elevation angle [14] as

$$
\sigma_{\text {recv }}(\mathrm{El})=0.15+0.43 \mathrm{e}^{\left(-\frac{\mathrm{El}}{6.9^{\circ}}\right)} .
$$

The code tracking error estimation model, taking into account the number of receiver design characteristics, can be found in [15].

- Multipath error estimation model mainly depends on the user environment. Airborne receivers will be subject to multipath from the airframe, ground, and other objects during the precision approach [16]. The airframe multipath is modeled [12] as

$$
\sigma_{\text {recv }}(\mathrm{El})=0.13+0.53 \mathrm{e}^{\left(-\frac{\mathrm{El}}{10^{\circ}}\right)} .
$$

Unlike aviation, multipath in an urban scenario can cause significant ranging errors due to a large number of reflectors. Modeling multipath in an urban scenario is a challenge mainly due to the dynamic nature of the user environment. An overbounding model for multipath error in an urban scenario is proposed in [9] for GNSS receivers that do not use advanced technology for multipath mitigation.

Estimating the pseudorange residual error standard deviation using the aforementioned models is referred to as the classical UERE method in this paper. A typical UERE budget as a function of satellite elevation angle, considering the classical UERE method, is presented in Fig. 1. We assume a single-frequency GPS user at a geomagnetic latitude of $48.18^{\circ}$, a carrier-tonoise power spectral density ratio of $45 \mathrm{dBHz}$, and a GPS URA value of $2.4 \mathrm{~m}$ to generate this figure.

\section{B. Issues of Using the Classical Residual Error Models in Urban User Environment}

The classical method of pseudorange residual error estimation is simple in its implementation and satisfies the integrity requirements of air navigation relying on the use of GNSS. However, the use of classical error models in estimating UERE and its subsequent use in deriving the positioning error bounds does not yield the required integrity and navigation system availability in the urban user environment, as demonstrated in

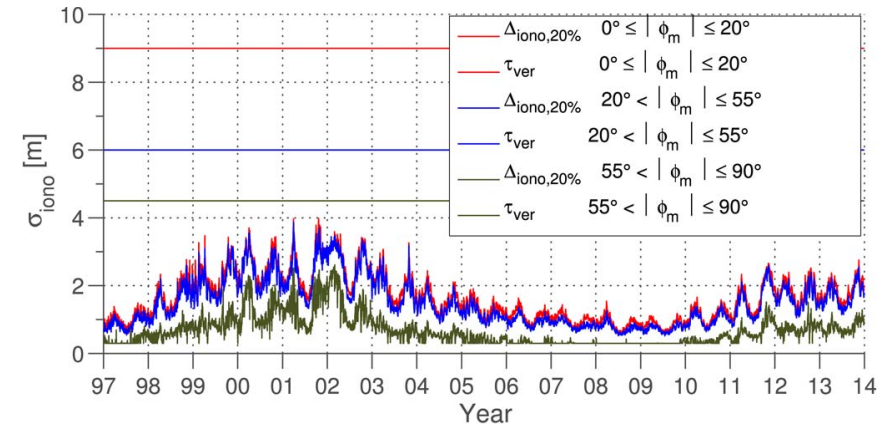

Fig. 2. Comparison of $\Delta_{\text {iono, } 20 \%}$ and $\tau_{\text {ver }}$.

[1]. This is due to the mismatch of error model (e.g., multipath) used in UERE estimation and the urban user environment. Equally contributing in degrading navigation system availability is the inherent conservatism, mainly in the broadcast URA and single-frequency ionospheric residual error models, which deters the navigation system availability by increasing the derived protection levels. These issues are discussed below.

- The pseudorange error budget for a single-frequency navigation user is dominated by the residual error due to ionosphere. The residual error model given in (4) is very conservative, as demonstrated in [9], using the historic data of daily maximum ionospheric correction. For the sake of completeness, we produced Fig. 2, taking inspiration from [9] while considering the Klobuchar-style ionospheric coefficients provided by Center of Orbit Determination in Europe [17]. It can be seen in Fig. 2 that the $\Delta_{\text {iono }}, 20 \%$ never reached the minimum vertical delay $\tau_{\text {ver }}$ over the period of 19972013. Therefore, (4) can be reduced to (11) as in [9]:

$$
\sigma_{\text {iono }}=F_{\mathrm{pp}} \cdot \tau_{\mathrm{ver}} .
$$

Equation (11) shows that $\sigma_{\text {iono }}$ provides a very conservative estimate of the residual error due to ionosphere.

- Broadcast URA values generated by the GPS operational and control segment are very conservative by design as they are computed by taking the root mean square (RMS) of instantaneous user range error values across the portion of the globe in view of the satellite to cover all the users that can view that particular satellite [11], [18]. The URA values, quantized to the level represented by a 4-bit index, are broadcast to the users through the navigation message. The URA index indicates the range of URA values, among which, the user has to use the maximum. This introduces a further degree of conservatism in the URA estimate used by the user [19].

- Multipath is a local phenomenon as it is exclusively based on the user environment. In an urban user scenario, multipath is very dynamic, which makes it extremely hard to model it. The error models for the estimation of multipath in urban environment [9] provides conservative estimation of the user environment, first due to overbounding that inflates the actual error variance and, second, due to the inherent design of the 
model that predicts the average behavior of the user environment. This results in increasing the protection levels, which ultimately degrades navigation system availability.

- Aviation receivers are sophisticated in design, and the receiver design characteristics are well studied, which provides the advantage of developing a suitable receiver error model. Unlike the aviation receiver, mass-market receivers are inexpensive, and the design characteristics are not known in most of the cases; therefore, estimating the pseudorange errors due to receiver noise and other imperfections for mass-market receivers is a challenge.

The inherent conservatism in broadcast URA and the singlefrequency ionospheric model and the mismatched multipath error model results in overly estimation of UERE, which ultimately contribute to the further degradation of the navigation system availability in urban environment. In order to improve the navigation system availability in urban environment, we propose a methodology that estimates UERE using raw pseudorange observables instead of relying on any theoretical and/or empirical error models in the estimation of UERE.

\section{Proposed Methodology}

The series of pseudorange values can be modeled as a discrete-time random process $\rho_{i}[\mathrm{n}]$ for each $i$ th satellite, where the stochastic component is due to the error introduced by the space segment, propagation, and the receiver processing. Such a process is not stationary due to the relative movement of the satellite with respect to the user. In order to get the deviation of each sample from its theoretical value in a run-time process implemented in the receiver, orbital models and estimation of biases due to the processing should be implemented. This solution would be unaffordable for mass-market receivers where computational complexity limitations and power saving are of paramount importance.

It has to be remarked that the pseudorange values are typically provided to the user at a rate of one value per second in most of the receivers. Not all the receivers output such a value, just providing the final estimated position. However, this work focuses on mass-market and professional receivers providing the raw pseudorange values, thus allowing further processing at the user level.

The proposed method for de-trending the measurements in order to remove the deterministic component of the satellite movement is a digital differentiation of the value series, thus highlighting the variability of the measurements with respect to the theoretical orbit. Being $\rho_{i}[n]$ the value of the measured pseudorange at the $n$th time epoch for the $i$ th satellite, the firstorder differentiation is operated as

$$
\rho_{i}^{(1)}[n]=\frac{1}{\sqrt{2}} \cdot\left(\rho_{i}[n+1]-\rho_{i}[n]\right) .
$$

The digital differentiation has then to be iterated until the variability due to the satellite orbit is totally removed. A first issue to address is the definition of the differentiation order $k$ to

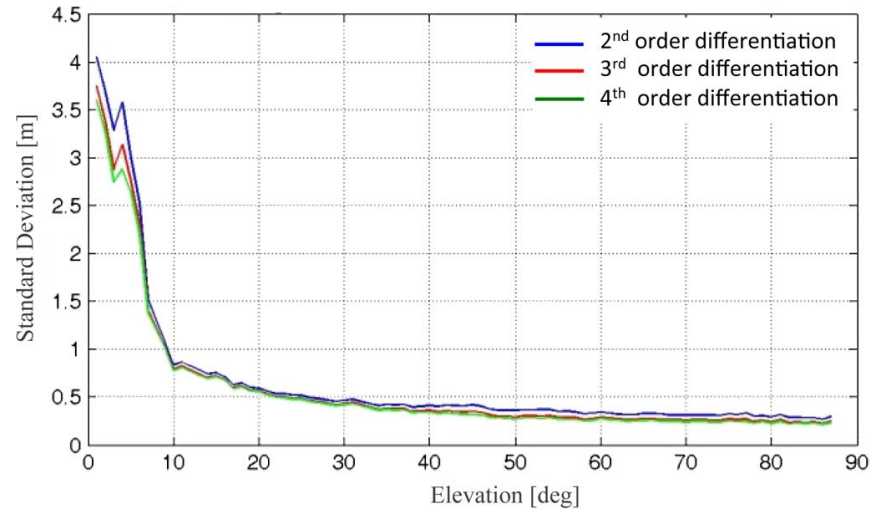

Fig. 3. Comparison of normalized results for second, third, and fourth differentiations for GPS PRN 1.

wipe off all the low-frequency components and to get a zeromean time series. The standard deviation can then be estimated on the basis of the differentiated data, taking into account a multiplication factor depending on the number of the computed differentiations. For first-order differentiation, the multiplication factor is $\frac{1}{\sqrt{2}}$, whereas for second- and third-order differentiations, the coefficient is $\frac{1}{\sqrt{6}}$ and $\frac{1}{\sqrt{20}}$, respectively.

However, this normalization is valid only if the samples taken at different time instants are uncorrelated. In fact, generally speaking, the standard deviation of a sum of Gaussian zeromean variables is

$$
\sigma_{x+y}^{2}=\sigma_{x}^{2}+\sigma_{y}^{2}+2 \cdot \operatorname{cov}(x, y)
$$

where, in this specific case, $x$ is the pseudorange $\rho_{i}[n]$ of the $i$ th satellite measured at time instant $n$, whereas $y$ is the pseudorange $\rho_{i}[n+1]$ of the $i$ th satellite measured at epoch $n+1$. The covariance is equal to zero only if the two variables are uncorrelated.

Fig. 3 shows the standard deviation obtained performing different higher-order differentiations for the GPS satellite PRN1. The data collection is performed using a mass-market receiver Ublox AEK-5T GPS and a geo-referenced antenna, in a static scenario in open sky where no multipath is likely present. It is clear that the standard deviation of the pseudorange value obtained for the second-order differentiation is sufficient to de-trend the data series, since no significant differences are obtained by the third or fourth orders. The difference in the estimated value is below $10 \%$ if third- and fourth-order differentiations are considered. Fig. 3 shows also that, as expected, when the satellite is at low elevations, it does not ensure the same quality of the signal, compared with higher elevations.

However, as previously anticipated, the estimation of $\sigma_{i}$ is valid if the considered samples are uncorrelated. It is likely that data measured at a rate of one sample per second do have correlated error contributions since the propagation conditions are basically the same. The differentiation operation may wipe off some of the error components, thus leading to overoptimistic estimations of $\sigma_{i}$.

In order to decrease the cross-correlation between pseudorange measurements taken at close time instants without threatening the reliability of the standard deviation estimation, 


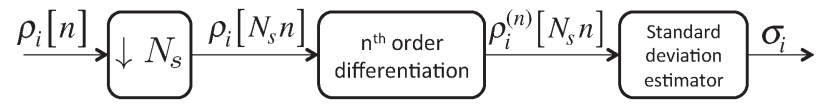

Fig. 4. Block diagram that shows the full processing chain for the run-time estimation of pseudorange standard deviation.

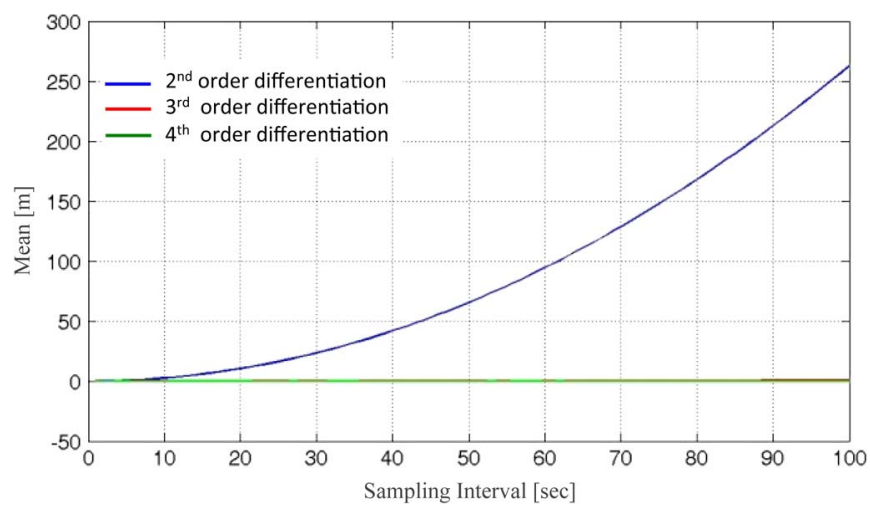

Fig. 5. Mean of pseudorange measurement for GPS PRN1.

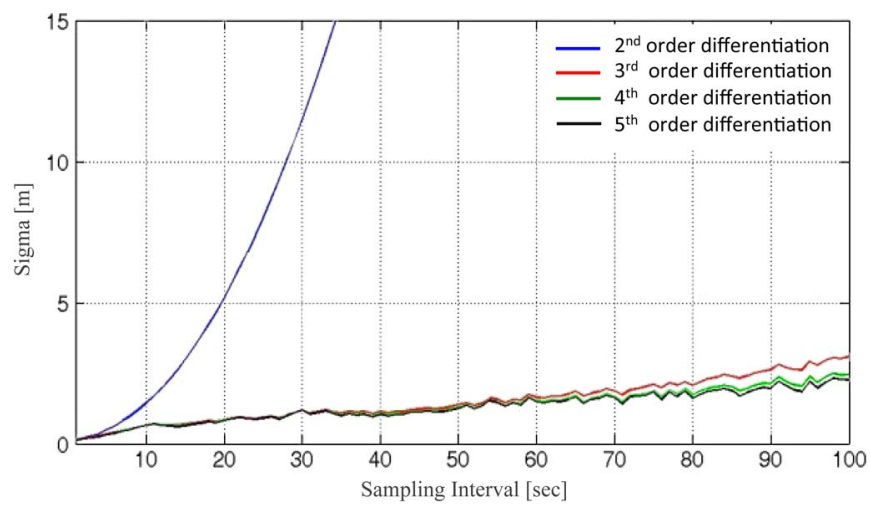

Fig. 6. Standard deviation of third- and fourth-order differentiations of the pseudorange measurement for PRN1.

an under sampling operation of $N_{s}^{i}$ values is implemented for the time series of values of the $i$ th satellite. If the samples of the measurements are selected with sufficient spacing in time, it is realistic to assume that the error contribution would not be correlated. Fig. 4 shows the full processing chain for the runtime estimation of the pseudorange error standard deviation. However, a tradeoff has to be identified, since if too many samples were discarded, the reliability of the estimated standard deviation would be threatened. The criterion to define the upper bound for $N_{s}^{i}$ has been based on the fact that the mean of values should always be zero. Fig. 5 shows the behavior of the mean considering the second-, third-, and fourth-order differentiations of PRN 1 and $1 \leq N_{s}^{i} \leq 100$.

From Fig. 5, it is clear that in increasing $N_{s}^{i}$, a second-order differentiation is not sufficient anymore. In fact, by increasing $N_{s}^{i}$, the mean rapidly increases as well. Considering samples taken at more distant time epochs, a third iteration is required in order to elide the mean component.

Fig. 6 shows the behavior of the standard deviations for $1 \leq N_{s}^{i} \leq 100$. The best sampling interval $N_{s}^{i}$ is computed as the maximum sampling period that ensures that the mean value of the third-order differentiation does not overcome the set value of 2.5. As a common value of the sampling interval $N_{s}$ for all the satellites in view, the median value of $N_{s}^{i}$ is chosen. The median is preferable with respect to the mean to remove the peak values caused by low elevation or a small number of available measurements for some satellites. On the basis of observables data collected over several hours by means of four different receivers (models and brands), the best sampling interval has been chosen empirically as $N_{s}=100$, corresponding in this case to values taken every $100 \mathrm{~s}$. It has to be remarked that such a choice depends on the test campaign performed using commercial receivers. The estimation algorithm aims at providing the estimation based on the output time series of the raw pseudorange values. Such values are correlated, not only for the slow variability of the environment, but also by the fact that the output value is the result of algorithms and, in some cases, of averaging strategies performed by the receivers software. Such strategies might introduce additional correlation among the values.

As a final check, in order to assure that the deviation of each sample from the theoretical value is only due to the error sources, the cross correlation between the time series (thirdorder differentiation and sampling time of $N_{s}=30$ ) belonging to different satellites has been assessed, and it was found to be negligible.

The major limitation of the proposed run-time method is the initial window period required for the estimation of UERE. Liability-critical applications relying on the use of GNSS need to wait initially (e.g., at least $100 \mathrm{~s}$ ) to obtain $\sigma_{\text {UERE }}$ estimate as demonstrated in this work.

\section{Performance of the Run-Time UERE ESTIMATION METHOD}

In order to assess the performance of the run-time method, we first compare UERE estimates obtained using the run-time and classical method; then, we compare the positioning accuracy obtained using run-time and classical UERE estimates, and finally, we compute the horizontal protection level (HPL) and analyze navigation system availability taking into account runtime and classical UERE estimates. To carry out performance assessment, code-based single-frequency pseudorange observables in a Receiver Independent Exchange file format [20] were collected using a Ublox AEK-5T GPS mass-market receiver placed in Turin, Italy at the Latitude: $45.07^{\circ}$, Longitude: $7.66^{\circ}$, and Altitude: $306.70 \mathrm{~m}$.

\section{A. Assessment of Estimated UERE}

UERE estimation of GPS PRN 6 using the classical and runtime UERE method is presented in Fig. 7. As shown, UERE estimated values using run time is lower than the classical method. To further extend our analysis to all satellites in view, we compare average, maximum, and minimum UERE, as shown in Figs. 8-10.

The average, maximum, and minimum run-time UERE is obtained by taking the average, maximum, and minimum UERE of all satellites in view. The resultant run-time UERE is then propagated in time for the next 100 epochs. 


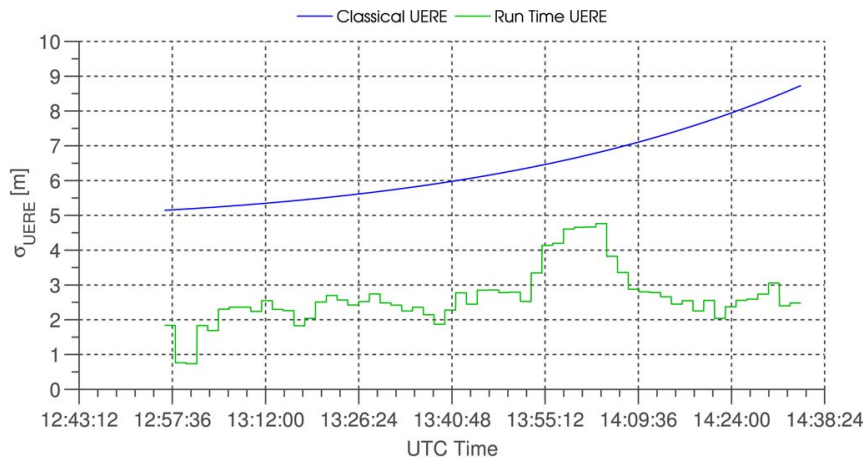

Fig. 7. UERE for GPS PRN 6 obtained using the classical and run-time UERE estimation method.

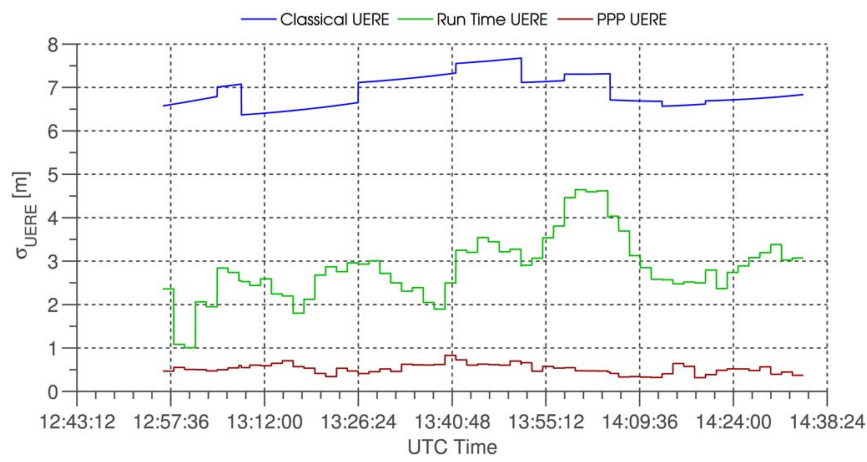

Fig. 8. Average UERE estimates of the classical and run-time UERE method.

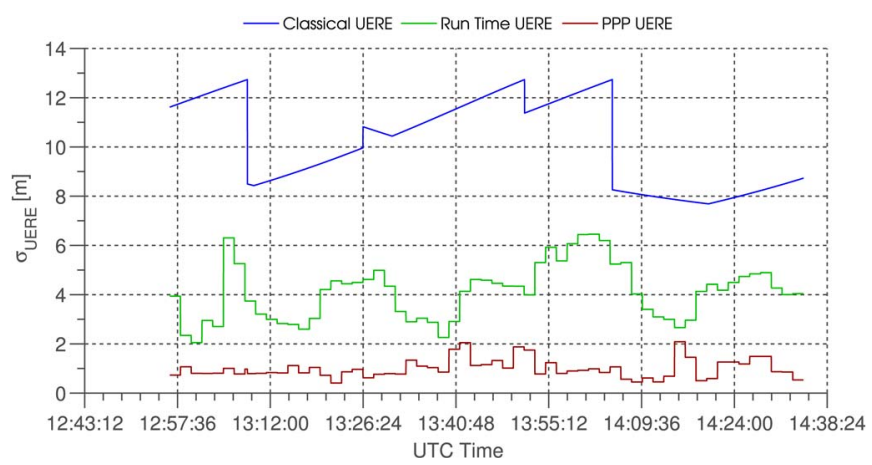

Fig. 9. Maximum UERE estimates of the classical and run-time UERE method.

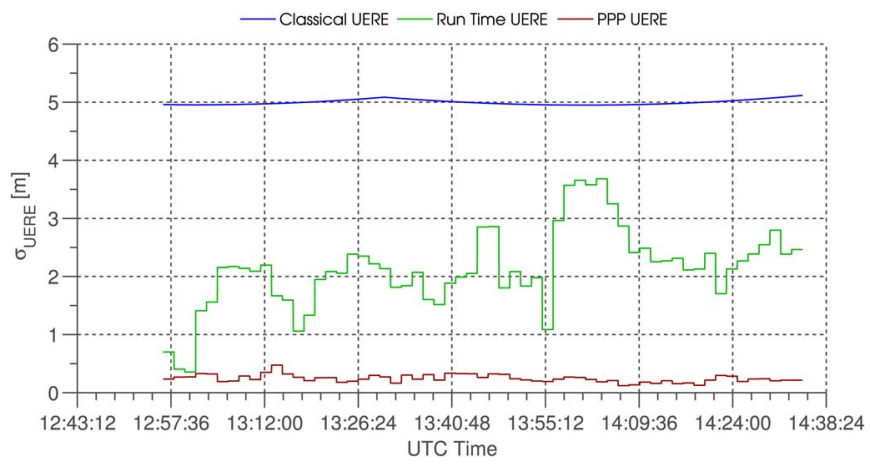

Fig. 10. Minimum UERE estimates of the classical and run-time UERE method.

The red curve represents the standard deviation of the pseudorange residuals obtained after applying single-frequency precise point positioning (PPP) correction models [21]. We obtain

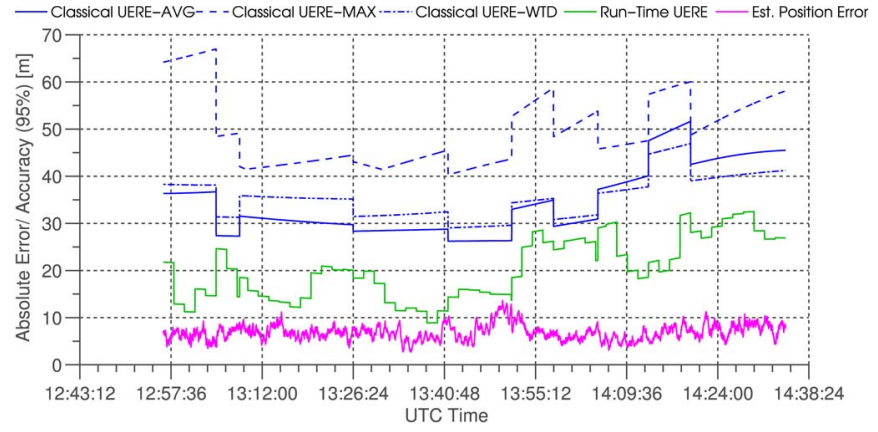

Fig. 11. Expected positioning accuracy (95\%) using classical and run-time UERE estimates. In the figure, MAX stands for maximum, AVG stands for average, and WTD stands for weighted.

the sample standard deviation over 100 epochs and then take the average over satellites in view, which represents the average standard deviation. The maximum standard deviation is obtained by taking the maximum over all satellites in view. PPP UERE is used as benchmark for performance comparison as it can be considered a minimum reliable estimation of UERE.

We obtain the average of classical UERE by taking the average over all the satellites in view at every epoch. The same goes for maximum and minimum UERE. Figs. 8-10 show that runtime UERE estimation of average, maximum, and minimum UERE is lower compared with classical UERE. This is mainly due to the fact that run-time UERE estimation is based on the perceived measurement errors according to the actual user environment. On the other hand, UERE estimation using the classical method is based on the residual error models that overbound the residual measurement errors to protect the worst-case user, for instance, single-frequency ionosphere residual error model [9]. The overbounding approach used by the classical method of UERE estimation results in conservative estimates, as shown in Figs. 8-10.

\section{B. Evaluation of Expected Positioning Accuracy}

The conservatism in the classical method of UERE estimation, when analyzed in the position domain, turns into a conservative estimate of the user positioning accuracy, as shown in Figs. 11 and 12. Estimated position error is the norm of the difference between surveyed position of the reference receiver and the estimated position obtained using single-frequency standard point positioning correction models. The estimated or expected positioning accuracy using run-time UERE estimate is obtained using (1) taking into account PDOP and HDOP, whereas $\sigma_{\text {UERE }}$ is taken as the maximum of all satellites in view. The expected positioning accuracy is compared with one obtained by means of the classical UERE estimate. Three different classical UERE estimates are used for the sake of comparison: weighted (WTD), maximum (MAX), and average (AVG). Averaging UERE might not be wise as it flattens the UERE estimate averaged over satellites at different elevation angles; however, after analyzing the performance of weighted and averaged UERE in terms of estimated positioning accuracy, it can be noticed that the performance of average and weighted UERE is approximately the same; therefore, it is decided to use average UERE estimate in the analysis for performance comparison. 


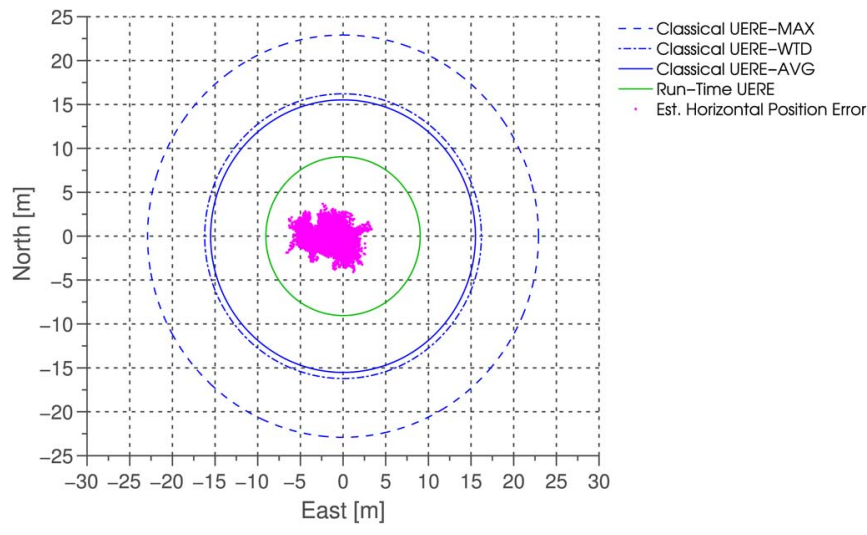

Fig. 12. Expected horizontal positioning accuracy (95\%) using classical and run-time UERE estimates.

The expected positioning accuracy using average and maximum UERE is obtained by (1), whereas the horizontal and 3-dimensional expected positioning accuracy using weighted UERE is obtained by (14) and (15), respectively, as in [22]. Thus

$$
\begin{aligned}
& \text { HRMS }=\sqrt{\left[\left(H^{T} \cdot W \cdot H\right)^{-1}\right]_{1,1}+\left[\left(H^{T} \cdot W \cdot H\right)^{-1}\right]_{2,2}} \\
& \text { PRMS }=\sqrt{\mathrm{HRMS}^{2}+\left[\left(H^{T} \cdot W \cdot H\right)^{-1}\right]_{3,3}} .
\end{aligned}
$$

HRMS is the 2-dimensional RMS expected accuracy in the horizontal dimension, PRMS is the 3-dimensional RMS expected accuracy in the horizontal and vertical dimension, $H$ is the geometry matrix, and $W$ is the inverse of the covariance matrix used for weighting of the satellites as is given by

$$
W=\left[\begin{array}{cccc}
\sigma_{\mathrm{UERE}, 1}^{2} & 0 & \cdots & 0 \\
0 & \sigma_{\mathrm{UERE}, 2}^{2} & \cdots & 0 \\
\vdots & \vdots & \ddots & \vdots \\
0 & 0 & \cdots & \sigma_{\mathrm{UERE}, \mathrm{i}}^{2}
\end{array}\right]^{-1}
$$

As shown in Figs. 11 and 12, the expected positioning accuracy using run-time UERE estimate provides a closer bound than that obtained using classical UERE estimates. This validates the impact of the run-time method on the expected positioning accuracy.

\section{Navigation System Availability}

Liability-critical services, which rely on the use of GNSS, require integrity monitoring of the GNSS signal [23], [24]. The navigation system provides integrity by comparing the computed protection level against a predefined alarm limit and declares the navigation system unavailable in case the protection level exceeds the predefined alarm limit. UERE is one of the factors that impact the protection levels, which are computed by the navigation user receiver using integrity monitoring methods. To analyze this impact, the HPL is computed taking into account classical and run-time UERE estimates using the solution separation receiver autonomous integrity monitoring (RAIM) technique [25]. Assuming the probability of false

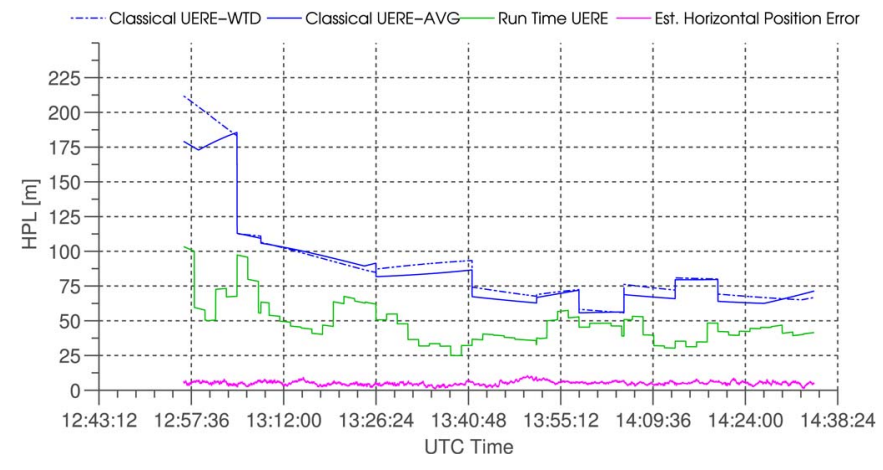

Fig. 13. RAIM HPL taking into account classical and run-time UERE estimates.

alarm $P_{f a}=5 \times 10^{-5}$ and probability of missed detection $P_{m d}=5 \times 10^{-5}$ as in [26], the HPL is computed as

$$
\begin{aligned}
& \mathrm{HPL}=\max \left\{\sqrt{d P_{n_{1,1}}+d P_{n_{1,2}}+d P_{n_{2,2}}} \cdot \mathrm{Q}^{-1}\left(\frac{P_{\mathrm{fa}}}{2 N}\right)\right. \\
& \left.+\sqrt{P_{n_{1,1}}+P_{n_{1,2}}+P_{n_{2,2}}} \cdot \mathrm{Q}^{-1}\left(\frac{P_{\mathrm{md}}}{N}\right)\right\} \text {. }
\end{aligned}
$$

$d P_{n}$ is the covariance matrix of the solution separation between the full set and $n^{\text {th }}$ subset solution, $P_{n}$ is the covariance of the $n^{\text {th }}$ subset solution, $N$ is the number satellites in view, and $Q^{-1}$ is the inverse of tail probability of standard Gaussian distribution:

$$
\begin{aligned}
d P_{n} & =\left(S_{0}-S_{n}\right) \cdot W^{-1} \cdot\left(S_{0}-S_{n}\right)^{T} \\
P_{n} & =S_{n} \cdot W^{-1} \cdot S_{n}^{T} \\
Q(x) & =\frac{1}{\sqrt{2 \pi}} \int_{x}^{\infty} e^{\frac{-t^{2}}{2}} d t .
\end{aligned}
$$

$S_{0}$ and $S_{n}$ are the least squares approximation of the full set and subset solution, respectively. It is worth mentioning that the focus here is to compare the respective HPLs not the performance of RAIM technique and the corresponding alarm and missed detection probabilities.

As shown in Fig. 13, tight HPL is derived using run-time UERE estimate as compared with classical UERE estimate. The protection levels derived using the conservative classical UERE estimate are too high as opposed to the protection levels derived by taking into account run-time UERE estimate. Assuming a horizontal alarm limit (HAL) of $60 \mathrm{~m}$, the navigation system availability is $83.44 \%$ taking into account run-time UERE estimate, whereas the navigation system availability is $7.13 \%$ using classical UERE estimate, as shown in Fig. 14. This shows that run-time UERE estimate significantly improves the navigation system availability by deriving tight HPL.

\section{CONCLUSION}

In this paper, a methodology for the run-time estimation of the pseudorange standard deviation has been proposed. The run-time UERE estimation method significantly increases navigation system availability by providing better approximation of the user environment. This extends the applicability of 


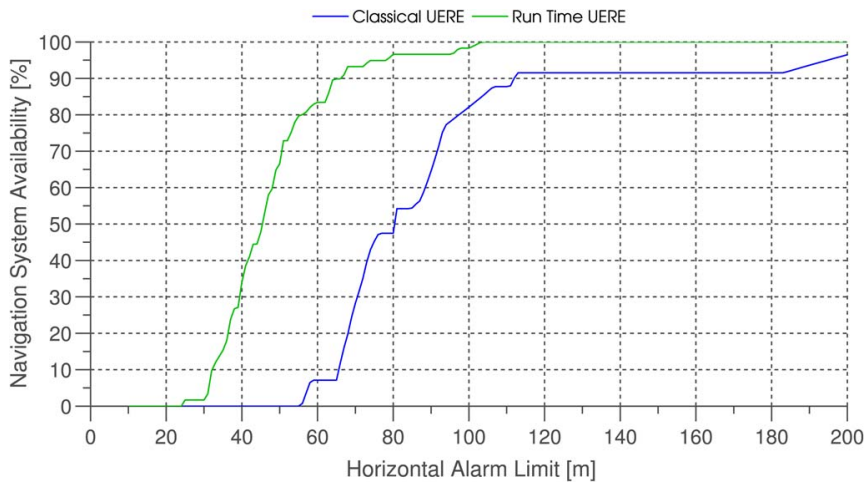

Fig. 14. Navigation system availability as a function of HAL.

GNSS in harsh environment (e.g., urban) without threatening the reliability of the obtained position solution. Furthermore, run-time UERE estimation provides the foundation for more elaborated processing both for accuracy and integrity that is required by a variety of liability-critical services relying on the use of GNSS. In addition, the advantage of the run-time method is an easy implementation since it avoids the use of orbital models in receiver for each satellite that would be necessary to remove the deterministic part of the measurement. It was shown how the estimated standard deviation provides a good approximation of the actual UERE and allows better estimation of the expected accuracy.

\section{REFERENCES}

[1] J. L. Leva, M. U. de Haag, and K. Van Dyke, "Performance of standalone GPS," in Understanding GPS: Principles and Applications. Norwood, MA, USA: Artech House, 1996, pp. 237-320, 1996.

[2] K. Ali, M. Pini, and F. Dovis, "Measured performance of the application of EGNOS in the road traffic sector," GPS Sol., vol. 16, no. 2, pp. 135-145, Apr. 2012.

[3] R. B. Langley, "Dilution of precision," GPS World, vol. 10, no. 5, pp. 52-59, 1999.

[4] M. Barros, L. Rosa, F. Walter, and L. Alves, "Global positioning system: A methodology for modelling the pseudorange measurements," Adv. Space Res., vol. 23, no. 8, pp. 1529-1532, 1999.

[5] Y. Koyama and T. Tanaka, "Improvements in accurate GPS positioning using time series analysis," in Proc. IEEE SICE, 2010, pp. 282-285.

[6] B. Belabbas, A. Hornbostel, and M. Z. Sadeque, "Error analysis of single frequency GPS measurements and impact on timing and positioning accuracy," in Proc. WPN/UET, 2005, pp. 81-86.

[7] B. Belabbas, F. Petitprez, and A. Hornbostel, "UERE analysis for static single frequency positioning using data of IGS stations," in Proc. Nat. Tech. Meet. Inst. Navig., 2001, pp. 310-319.

[8] B. De Cleene, "Defining pseudorange integrity-overbounding," in Proc. 13th Int. Tech. Meet. Satell. Div. ION GPS, 2000, pp. 1916-1924.

[9] D. Salós, C. Macabiau, A. Martineau, B. Bonhoure, and D. Kubrak, "Nominal GNSS pseudorange measurement model for vehicular urban applications," in Proc. IEEE/ION PLANS, 2010, pp. 806-815.

[10] Navstar GPS Space Segment/Navigation User Interfaces, Interface Specification, Global Positioning System Directorate, System Engineering and Integration Interface Specification, Los Angeles, CA, USA, IS-GPS-200, 2012.

[11] L. Heng, G. X. Gao, T. Walter, and P. Enge, "Statistical characterization of GPS signal-in-space errors," in Proc. ION ITM, San Diego, CA, USA, 2011, pp. 312-319.

[12] Minimum Operational Performance Standards for Global Positioning System/Wide Area Augmentation System Airborne Equipment, RTCA/ D0-229D SC-159, RTCA, Inc., Dec. 13, 2006.

[13] J. Klobuchar, "Ionospheric time-delay algorithms for single-frequency GPS users," IEEE Trans. Aerosp. Electron. Syst., vol. AES-23, no. 3, pp. 325-331, May 1987.

[14] G. A. McGraw, T. Murphy, M. Brenner, S. Pullen, and A. J. V. Dierendonck, "Development of the LAAS accuracy models," in Proc. 13th Int.
Tech. Meet. Satell. Div. ION GPS, Salt Lake City, UT, USA, 2000, pp. 1212-1223.

[15] P. W. Ward, J. W. Betz, and C. J. Hegarty, "Satellite signal acquisition, tracking, and data demodulation," in Understanding GPS: Principles and Applications, 2nd ed., E. D. Kaplan, and C. J. Hegarty, Eds. Norwood, MA, USA: Artech House, ch. 5, pp. 194-199.

[16] T. Murphy, R. Snow, and M. Braasch, "GPS multipath on large commercial air transport airframes," Navigation, vol. 43, no. 4, pp. 397-406, Winter 1996.

[17] R. Neilan et al., "IGS technical report 2013," Int. GNSS Serv. Central Bureau, Pasadena, CA, USA, Tech. Rep., May 2014.

[18] Global Positioning System Standard Positioning Service Performance Standard, US DoD, 2008.

[19] S. Pullen et al., "The impact of GPS modernization on standalone user performance and integrity with ARAIM," in Proc. 26th Int. Tech. Meet. Satell. Div. ION GNSS+, Sep. 2013, pp. 2637-2653.

[20] W. Gurtner, and L. Estery, "Rinex-the receiver independent exchange format-version 3.00," Astron. Inst., Univ. Bern UNAVCO, Boulder, CO, USA, 2007.

[21] Y. Mireault, P. Tétreault, F. Lahaye, P. Héroux, and J. Kouba, "Online precise point positioning," GPS World, vol. 19, no. 9, pp. 59-64, Sep. 2008.

[22] Todd Walter and Per Enge, "Weighted RAIM for precision approach," in Proc. ION GPS 8th Int. Tech. Meet. Satell. Div., Palm Springs, CA, USA, Sep. 12-15, 1995, pp. 499-509.

[23] J. Cosmen-Schortmann, M. Azaola-Saenz, M. A. Martinez-Olague, and M. Toledo-Lopez, "Integrity in urban and road environments and its use in liability critical applications," in Proc. IEEE/ION Position, Loc. Navig. Symp., 2008, pp. 972-983.

[24] T. Beech, M. Martinez-Olague, and J. Cosmen-Schortmann, "Integrity: A key enabler for liability critical applications," in Proc. 61st Аnпи. Meet. Inst. Navig., 2001, pp. 1-10.

[25] M. Brenner, "Integrated GPS/inertial fault detection availability," Navigation, vol. 43, no. 2, pp. 111-130, Summer 1996.

[26] D. Salós, A. Martineau, C. Macabiau, B. Bonhoure, and D. Kubrak, "Receiver autonomous integrity monitoring of GNSS signals for electronic toll collection," IEEE Trans. Intell. Transp. Syst., vol. 15, no. 1, pp. 94103, Feb. 2014

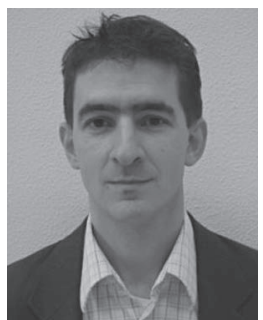

Fabio Dovis is an Associate Professor with the Department of Electronics and Telecommunications, Politecnico di Torino, Turin, Italy, as a member of the Navigation Signal Analysis and Simulation (NavSAS) group. He has relevant experience in European projects in satellite navigation as well as cooperation with industries and research centers. His research interests cover the design of GPS and Galileo receivers and advanced signal processing for interference and multipath detection and mitigation.

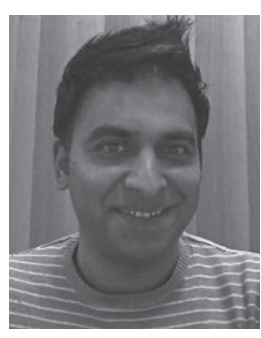

Bilal Muhammad is currently working toward the $\mathrm{Ph}$.D. degree in the Ph.D. School of Microelectronics and Telecommunication Engineering, University of Rome Tor Vergata, Rome, Italy.

$\mathrm{He}$ is working on satellite navigation services for civil aviation, which is a joint partnership of the Italian Space Agency and the ENAV Italian Authority for the provision of air traffic services. His research interests include the design and performance analysis of advanced receiver autonomous integrity monitoring architectures and the impact of space weather on the performance of GNSS receivers. 


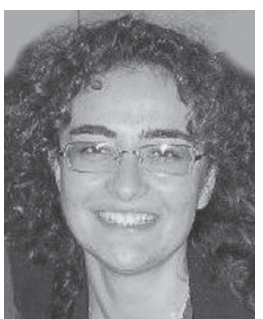

Ernestina Cianca is an Assistant Professor with the Department of Electronic Engineering, University of Rome Tor Vergata, Rome, Italy, and a member of the Interdisciplinary Center for TeleInfrastruktur. She is a Coordinator of the Master in Advanced Satellite Communication and Navigation Systems. She has relevant experience in National and European projects. In particular, she has been involved in several NavCom projects. She has published about 80 papers on international journal and conference proceedings. Her research interests cover wireless access technologies (code-division multiple access, orthogonal frequencydivision multiplexing, and single-carrier frequency-domain equalization) and equalization for both terrestrial and satellite communications, satellite communications in EHF bands (in particular, W-band), and integrity in GNSS systems.

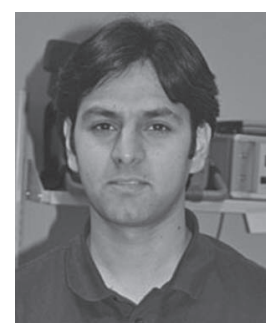

Khurram Ali received the B.Sc. degree in electrical engineering from the University of Engineering and Technology, Lahore, Pakistan, in 2007 and the Master's degree in navigation and related applications and the Ph.D. degree in electronics and communications from Politecnico di Torino, Turin, Italy, in 2009 and 2013, respectively.

His Ph.D. research thesis work was focused on the reliability of GNSS positioning in urban environment, specifically addressing the problems related to the road user sector. His research interests include interference and threats to future navigation systems, receiver technologies for mass-market applications, signal processing for GNSS receivers, and growth and future development of GNSSs and location-based services. 